\title{
Aspectos éticos relacionados ao processo de comunicação efetiva durante pandemia COVID-19: revisão integrativa
}

RESUMO | Objetivo: Investigar a produção de literatura sobre a comunicação da equipe de saúde com pacientes e familiares durante a pandemia da COVID-19. Método: Trata-se de uma revisão integrativa realizada nas bases de dados BVS, PubMed e Scopus, utilizando os descritores em ciências da saúde (DeCS): Coronavirus, Bioética e Comunicação. Resultados: Obtiveram-se 139 artigos, sendo selecionados 6 artigos para análise dessa revisão. Os achados mais comuns foram: os desafios no processo de comunicação, principalmente entre populações mais vulneráveis, como a oncologia, cuidados paliativos e pacientes de unidades de terapia intensiva; na outra ponta estratégias que visam mitigar esse agravante, como métodos alternativos de comunicação, que inclue um componente de vídeo. Conclusão: A comunicação efetiva ficou comprometida durante a pandemia da COVID-19, sendo necessário a criação de protocolos com base em parâmetros éticos e bioéticos conforme os valores de cada sociedade.

Palavras-chaves: Coronavírus; Comunicação; Bioética.

ABSTRACT | Objective: Investigate the production of literature on the communication of the health team with patients and families during the COVID-19 pandemic. Method: This is an integrative review carried out in the VHL, PubMed and Scopus databases, using the health science descriptors (DeCS): Coronavirus, Bioethics and Communication. Results: 139 articles were obtained, with 6 articles selected for analysis of this review. The most common findings were: challenges in the communication process, especially among the most vulnerable populations, such as oncology, palliative care and intensive care unit patients; at the other end, strategies aimed at mitigating this problem, such as alternative methods of communication, which include a video component. Conclusion: Effective communication was compromised during the COVID-19 pandemic, requiring the creation of protocols based on ethical and bioethical parameters according to the values of each society.

Keywords: Coronavirus; Communication; Bioethics.

RESUMEN | Objetivo: Investigar la producción de literatura sobre la comunicación del equipo de salud con pacientes y familiares durante la pandemia COVID-19. Método: Se trata de una revisión integradora realizada en las bases de datos BVS, PubMed y Scopus, utilizando los descriptores de ciencias de la salud (DeCS): Coronavirus, Bioética y Comunicación. Resultados: Se obtuvieron 139 artículos, con 6 artículos seleccionados para el análisis de esta revisión. Los hallazgos más comunes fueron: desafíos en el proceso de comunicación, especialmente entre las poblaciones más vulnerables, como pacientes de oncología, cuidados paliativos y unidad de cuidados intensivos; en el otro extremo, estrategias destinadas a mitigar este problema, como los métodos alternativos de comunicación, que incluyen un componente de video. Conclusión: La comunicación efectiva se vio comprometida durante la pandemia de COVID-19, requiriendo la creación de protocolos basados en parámetros éticos y bioéticos acordes a los valores de cada sociedad.

Palabras claves: Coronavirus; Comunicación; Bioética.

\section{Bruna Caroline Silva Falcão}

Enfermeira. Universidade Federal do Maranhão. Mestranda do Programa de Pós Graduação em Enfermagem.

ORCID: 0000-0001-50281670

\section{Jocelha Maria Costa de Almeida}

Enfermeira. Especialista em Saúde da Família e Unidade de Terapia Intensiva. Mestranda do Mestrado Acadêmico em Enfermagem da Universidade Federal do Maranhão.

ORCID: 0000-0002-3147-954X

\section{Adriana Torres dos Santos}

Enfermeira. Mestranda do Mestrado Acadêmico em Enfermagem da Universidade Federal do Maranhão.

ORCID: 0000-0002-8415-0879

\section{Elza Lima da Silva}

Doutora em Ciências Médicas. Enfermeira. Doutora em Ciências Médicas. Universidade Federal do Maranhão.

ORCID: 0000-0002-0287-046X

\section{Nair Portela Silva Coutinho}

Enfermeira. Doutora em Ciências da Saúde. Universidade Federal do Maranhão. ORCID: 0000-0002-2050-026X

\section{Lena Maria Barros Fonseca}

Enfermeira. Doutora em Biotecnologia pela Rede Nordeste de Biotecnologia. Universidade Federal do Maranhão. ORCID:0000-0002-6499-1005

Recebido em: 30/11/2020

Aprovado em: 02/02/2021
INTRODUÇÃO

$\Lambda$ epidemia da COVID-19 teve início em janeiro de 2020, quando em um curto espaço de tempo atingiu vários países, sendo considerado pandemia em 11 de março de 2020 pela Organização Mundial de Saúde (OMS). A elevada disseminação do vírus, a taxa de mortalidade que varia de 2 a $15 \%$, o elevado número de casos e a evolução rápida dos casos graves, ocasionou um aumento acentuado das internações hospitalares, da utilização dos recursos de terapia intensiva e de mortes ${ }^{1}$.

A comunicação efetiva tornou-se um desafio na pandemia devido às medidas sanitárias instaladas para controle da dis- 
seminação do vírus. No entanto, estratégias são necessárias para assegurar que metas internacionais de segurança do paciente sejam desenvolvidas ${ }^{2}$. Outro desafio tem sido conservar os princípios da bioética, principalmente quando se fala em isolamento social onde o princípio da justiça pode limitar o da autonomia, porém há de se concordar que o utilitarismo deve ser a aplicado, ou seja fazer o bem para o maior número de pessoas, e isso envolve restrição e medidas sanitárias ${ }^{3}$.

A comunicação envolve a relação entre uma pessoa e outra, seja presencial ou virtual, nesse sentido é possível manter pacientes em ambientes de enfermaria ou intensivo com seus próprios aparelhos telefônicos, sendo sugerido o uso do aplicativo WhatsApp para viabilizar visitas, é necessário ainda montar uma equipe de comunicação compatível com a demanda de cuidados e ser referência para o paciente ${ }^{1}$.

A comunicação de más notícias, em especial a comunicação do óbito, pode se tornar necessária e frequente no cenário da COVID-19 e exige uma flexibilização da circunstância, de forma individualizada, pois a maneira como as informações são transmitidas podem influenciar na forma de enfrentamento do conteúdo comunicado ${ }^{4,5}$.

O Conselho Federal de Medicina por meio do Parecer no 14/2017 reconhece o WhatsApp como importante ferramenta de comunicação entre médicos em caráter privativo para o repasse de informações confidenciais inerentes ao estado clínico de pacientes para enviar dados ou tirar dúvidas, a plataforma também pode ser usada para comunicação entre médico e paciente ${ }^{6}$.

Dessa forma, a pesquisa justifica-se pela necessidade de levantamento sobre o que vem sendo estudado e realizado referente a comunicação com pacientes e familiares durante a pandemia frente ao isolamento dos usuários, visto que a comunicação efetiva leva uma assistência segura, de qualidade e com custos reduzidos. Nesse sentido, considerando o distancia- mento que familiares tiveram que obedecer deixando seus entes queridos aos cuidados da equipe hospitalar, esta pesquisa objetivou responder a seguinte questão: o que as produções científicas apontam sobre a comunicação efetiva da equipe de saúde, pacientes e familiares durante a pandemia pelo novo coronavírus?

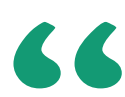

\section{Outro desafio tem sido conservar os princípios da bioética, principalmente quando se fala em isolamento social onde o princípio da justiça pode limitar o da autonomia...}

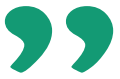

\section{MÉTODO}

O estudo trata-se de uma revisão integrativa, a escolha da temática permite a análise de pesquisas relevantes por meio da realização de busca e a avaliação crítica das principais evidências. Este método busca a exaustão de estudos referente ao tema investigado por meio da seleção justificada de estudos, utilizando critérios específicos de inclusão e exclusão. A revisão integrativa proporciona a síntese de conhecimento e a aplicabilidade dos resultados de estudos significativos na prática $^{7,8}$

A pesquisa seguiu as seguintes etapas: identificação do tema de investigação e elaboração da questão de pesquisa; estabelecimento dos critérios de inclusão e exclusão dos estudos; avaliação e interpretação dos resultados e apresentação dos dados na estruturação de revisão integrativa ${ }^{9}$.

Na primeira etapa da pesquisa, buscou-se definir o tema a ser investigado, para isso foi elaborada a seguinte questão de pesquisa: $O$ que as produções científicas têm evidenciado sobre os aspectos éticos relacionados a comunicação efetiva da equipe de saúde, pacientes e familiares durante a pandemia da COVID-19?

Foi estabelecido como critérios de inclusão os estudos disponíveis eletronicamente, nos idiomas português, inglês ou espanhol e que abordassem a temática do processo de comunicação durante a pandemia da COVID-19, não foi estabelecido critérios de anos para as publicações pois a temática do estudo é referente a pandemia da COVID-19, sendo todos os estudos publicados no ano de 2020.

A busca ocorreu no mês de dezembro de 2020, em três bases de dados: PUBMED onde foram encontrados 24 artigos, BVS onde foram encontrados 99 artigos e SCOPUS onde foram encontrados 16 artigos. Os termos utilizados para o levantamento dos artigos foram extraídos dos Descritores em Ciências da Saúde (Decs) e do Medical Subject headings, (MeSH), sendo utilizado uma combinação dos seguintes descritores: "bioethics", "communication", "coronavírus" com a associação de descritores boolenos OR e AND.

A seleção dos foi realizada em 3 etapas: a primeira foi realizada uma busca ampla dos artigos a partir da combinação dos descritores e aplicação dos critérios de inclusão e exclusão, sendo identificados um total de 139 artigos.

$\mathrm{Na}$ segunda fase, todo o material levantado foi submetido ao gerenciador bibliográfico Mendeley, a ferramen- 
ta possibilitou a exclusão de 25 artigos duplicados. Posteriormente foi realizada

a leitura crítica e reflexiva dos títulos e resumos de 114 artigos, após essa fase fo-

\section{Figura 1. Fluxograma de construção do corpus da pesquisa. São luís - MA, Brasil, 2020.}

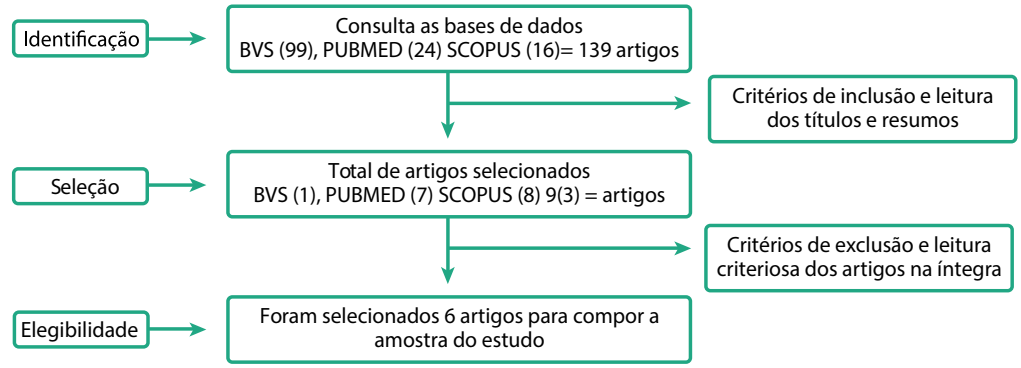

ram selecionados 19 artigos para leitura criteriosa e na íntegra.

$\mathrm{Na}$ terceira etapa, denominada de elegibilidade foi construído um instrumento próprio em forma de planilha eletrônica no Excel que orientou quais informações seriam extraídas dos estudos selecionados, com o objetivo de reunir e sintetizar as informações coletadas nos artigos, o instrumento foi preenchido com as seguintes informações: título, autor, ano de publicação, periódico, objetivos e resultados da pesquisa. Em seguida, foram selecionados 6 artigos que constituíram o corpus da pesquisa, conforme demonstrado na Figura 1.

Fonte: Dados da pesquisa.

\section{Quadro 1. Distribuição dos artigos caracterizadas pelo título, autores e ano, base de dados e resultados. São Luís - MA, Brasil, 2020.}

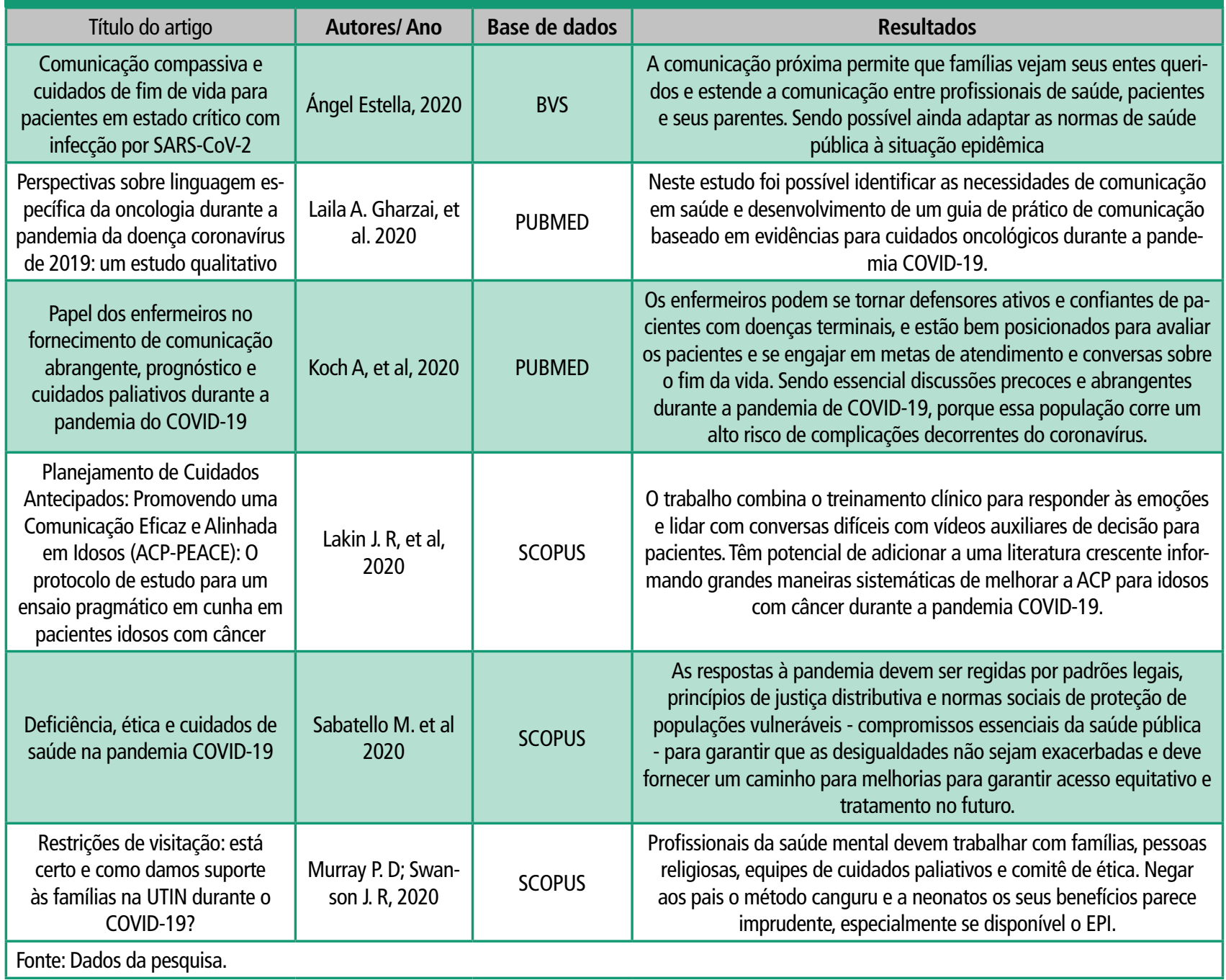




\section{RESULTADOS}

A descrição dos artigos que compuseram a revisão integrativa encontra-se no Quadro 1, contendo o título, autor, ano de publicação, base de dados científicas responsável pela indexação e os principais resultados que trouxeram contribuições para responder à questão de estudo e emergiram duas categorias temáticas: $\mathrm{O}$ processo de comunicação efetiva durante a pandemia da COVID-19 e Estratégias para melhorar a comunicação durante a pandemia COVID-19.

O processo de comunicação durante a pandemia da COVID-19.

A doença causada pelo novo coronavírus (Covid-19) é atualmente um problema mundial de saúde pública. Muitos oncologistas estão tendo conversas desafiadoras com seus pacientes sobre como a pandemia de COVID-19 está afetando o tratamento do câncer e podem desejar orientação de comunicação baseada em evidências ${ }^{10}$.

Um estudo identificou cenários específicos de oncologia relacionados a $\mathrm{CO}$ VID-19 onde a comunicação foi desafiadora, na maioria dos cenários, as reações do paciente envolveram raiva, medo e ansiedade. Os pacientes e suas famílias devem, portanto, receber informações precisas desde o início da doença e serem disponibilizadas possibilidades de suporte emocional, espiritual e social ao longo de todo o curso da doença e após a morte do paciente, a partir do acompanhamento do luto ${ }^{11}$.

Outro estudo confirmou ser importante que enfermeiros de cuidados paliativos promovam e mantenham discussões precoces e abrangentes durante a pandemia de COVID-19, principalmente para as populações que possuem um risco maior de complicações decorrentes do coronavírus. $\mathrm{O}$ isolamento dos pacientes nos hospitais e as restrições de visitas impõem às famílias profundas transformações na forma de cuidar e conceber o cuidado para com seus entes queridos, dificultando a com- preensão acerca da finitude da vida e das mudanças a serem enfrentadas pela morte de alguém próximo ${ }^{11,12}$.

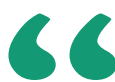

Muitos oncologistas
estão tendo conversas desafiadoras com seus pacientes sobre como a pandemia de COVID-19 está afetando o tratamento do câncer e podem desejar orientação de comunicação baseada em evidências.

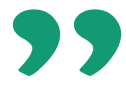

Estratégias para melhorar a comunicação durante a pandemia COVID 19.

Um dos estudos realizados comprovou que do ponto de vista ético, a restrição à visitação dos familiares em ambientes hospitalares é pouco compreensível, independentemente do método de inclusão, o papel da família à beira do leito, mesmo que virtual, é de suma importância. Os autores identificaram ainda métodos alternativos de comunicação que incluem a videoconferência baseada na web como o FaceTime e Skype e sistemas de câmera comercialmente disponíveis ${ }^{13}$.

Em outro estudo, os autores buscaram métodos e a justificativa de um ensaio que visa melhorar o planejamento antecipado de cuidados para pacientes idosos com câncer avançado e o protocolo modificado em resposta às mudanças trazidas pela pandemia COVID-19. O trabalho combina o treinamento clínico para responder às emoções e lidar com conversas difíceis com vídeos de auxílio à decisão para os pacientes ${ }^{14}$.

Existe uma aplicação limitada das estruturas existentes de planejamento de emergência com e para pessoas com deficiência na pandemia COVID-19, é necessário explorar as preocupações que afetam os cuidados de saúde das pessoas com deficiência e indicar as soluções possíveis. Medidas clínica e políticas de saúde pública são essenciais para garantir que pessoas com deficiência sejam incluídas no planejamento de esforços futuros relacionados à pandemia. Para os autores a devastação provocada pela pandemia COVID-19 levanta dilemas desafiadores em bioética ${ }^{15}$.

Além disso, questões de justiça social atormentaram comunidades historicamente marginalizadas nos Estados Uni$\operatorname{dos}^{13}$. As respostas à pandemia devem ser regidas por padrões legais, princípios de justiça distributiva e normas sociais de proteção de populações vulneráveis - compromissos essenciais da saúde pública - para garantir que as desigualdades não sejam exacerbadas e deve fornecer um caminho para melhorias para garantir acesso equitativo e tratamento no futuro ${ }^{15}$.

\section{DISCUSSÃO}

De fato, o processo de comunicação tem como papel primordial garantir o repasse de informações verbais precisas e completas referentes à assistência de saúde prestada ao paciente, e para que ocorra de forma eficiente é fundamental a empatia entre os membros envolvidos no processo, assim como a certificação que a comunicação de fato ocorreu, assim a família sente segurança na conduta profissional e no cuidado prestado ${ }^{2}$. 
Em uma pesquisa realizada decidiu-se que a comunicação de óbito de pacientes seria realizada via ligação telefônica, e chamou atenção a falha na formação do profissional, observou-se que os médicos são responsáveis por comunicar $46,2 \%$ das notícias de óbito e apenas $7,7 \%$ referiram que participaram de treinamentos práticos e eficientes sobre essa temática ${ }^{16}$.

Para estabelecer condutas bioéticas na pandemia, é essencial conhecer o ponto de vista da família e paciente, pensando nisso, um estudo desenvolveu um protocolo de critérios de gravidade para COVID-19 e incluiu uma consulta ao paciente e familiar para esclarecer desejos e dúvidas referentes ao fim de vida, levantando os níveis de prioridade para esse momento tão sofrido ${ }^{17}$.

A pandemia fez os serviços de saúde reinventar formas alternativas de repasse de informações implantação urgente de soluções tecnológicas para a comunicação entre pacientes e familiares incentivando a realização de chamadas de vídeo entre pacientes e suas famílias

6

observou-se que os médicos são responsáveis por comunicar $46,2 \%$ das notícias de óbito e apenas $7,7 \%$ referiram que participaram de treinamentos práticos e eficientes sobre essa temática e uso da realidade virtual imersiva para aproximar a experiência do contato e promover uma assistência de saúde de qualidade $\mathrm{e}^{16,18}$.

\section{CONCLUSÃO}

Fica claro que o relacionamento interpessoal com pacientes e familiares é uma ferramenta imprescindível à promoção de cuidados, pois é o momento que permite o esclarecimento de dúvidas com uma linguagem simples e acessível e oportuniza manifestarem suas angústias com a equipe assistencial.

A bioética fornece meios para a tomada de decisão difíceis, mas isso não quer dizer que a bioética impõe a decisão. Percebe-se que a comunicação sobre o estado de saúde dos pacientes ficou comprometida, tanto no que tange esse diálogo com a família e o próprio paciente. É preciso que sejam criadas mais diretrizes para sanar esse impeditivo com base em parâmetros éticos e bioéticos, escolhidos de acordo com os valores de cada sociedade. 2

\section{Referências}

1. Crispin DH, Silva MJP, Cedotti W, CÂMARA M, Gomes SA. Comunicação difícil e COVID-19: Recomendações práticas para comunicação e acolhimento em diferentes cenários da pandemia; 2020.

2. Olino, Luciana et al. Comunicação efetiva para a segurança do paciente: nota de transferência e Modified Early Warning Score. Rev Gaúcha Enferm. 2019; 40(esp):e20180341.

3. Sanches, MA, Cunha TR, Siqueira SS, Siqueira JE. Perspectivas bioéticas sobre tomada de decisão em tempos de pandemia. Rev. Bioét. 2020. 28 (3).

4. Paes da Silva MJ. Comunicando Más Notícias. 0 Mundo da Saúde. 2012;36(1):49-53.

5. Maria E, Leitão P. A comunicação de más notícias: mentira piedosa ou sinceridade cuidadosa. A Comun más notícias mentiram piedosa ou sinceridade Cuid. 2012;11(2):58-62.

6. Conselho Federal de Medicina (Brasil). Parecer 14/2017. Dispõe sobre o uso de WhatsApp em ambiente hospitalar. Disponível em https://sistemas.cfm.org. br. Acesso em: 30 dez. 2020

7. Mendes KDS, Silveira RCCP, Galvão CM. Revisão integrativa: método de pesquisa para a incorporação de evidências na saúde e na enfermagem. Texto contexto - enferm. 2008; 17 (4): 758-764.

8. Souza, M. T.; Silva, M. D.; Carvalho, M. D. Integrative review: what is it?: how to do it?. Einstein, São Paulo, v. 8, n. 1, p. 102-106, 2010.

9. Marinus, M. W. L. C; Queiroga, B. A. M; Moreno, L, R; Lima, S. R; Comunicação nas práticas em saúde: revisão integrativa da literatura. Sáude Soc. São Paulo, v.23, n.4, p.1356-1369, 2014.

10. Dantas FJ, et. al. Covid-19 e Câncer: Atualização de Aspectos Epidemiológicos. Rev. Bras. Cancerol. [Internet]. 14 $4^{\circ}$ de maio de 2020 [citado 01 de abril de 2021];66(TemaAtual):e-1013. Disponível em: https://rbc.inca.gov.br/revista/ index.php/revista/article/view/1013

11. Romanò M. Fra cure intensive e cure palliative ai tempi di CoViD-19. Recenti Prog Med. 2020; 111(4):223-30.

12. Koch A, Mnatzomis S. Nurses' Role in Providing Comprehensive Communication, Prognostication, and Palliative Care During the COVID-19 Pandemic. J. Hosp. Palliat Nurs. 2020:22(6): 442-446.

13. Murray D, Swanson JR. Visitation restrictions: is it right and how do we support familiesin the NICU during COVID-19? Journal of Perinatology, v. 40, p. 1576-1581, 2020. DOI: 0.1038/s41372-020-00781-1.

14. Lakin JR, Brannen EN, Tulsky JA, Paasche-Orlow MK, Lindvall C, Chang Y, Gundersen DA, El-Jawahri A. Advance Care Planning: Promoting Effective and Aligned Communication in the Elderly (ACP-PEACE): the study protocol for a pragmatic stepped-wedge trial of older patients with cancer. BMJ Open, v. 10, n.7, 2020. DOl: 10.1136 .

15. Sabatello M, Blankmeyer B, McDonald K.E, Appelbaum PS. Disability, EthiCS, and Health Care in the COVID-19 Pandemic. American Public Health Association. 2020: 110:1523-27. DOI: 10.2105/AJPH.2020.305837.

16. Alcantara, Elen Resende de Almeida, et al. Treinamento para comunicação de óbito durante a pandemia COVID-19. Revista Qualidade HC.

17. Batista RS, Gomes AP, Braga LM. Covid 19 e o fim da vida: Quem será admitido na unidade de terapia intensiva. Observatório COVID19. Informação em ação. 2020.

18. SOUSA JBA et al, Comunicação efetiva como ferramenta de qualidade: Desafio na segurança do paciente. Curitiba. v. 3, n. 3, p.6467-6479 may./jun. 2020. 